\title{
PREDiction OF Wear of WOOdWORKING TOOLS DEPENDING OF THE CHIP THICKNESS BY BOUNDARY ELEMENT METHOD SIMULATION
}

\author{
Ibrahim Busuladžić, Izet Horman, Seid Hajdarević
}
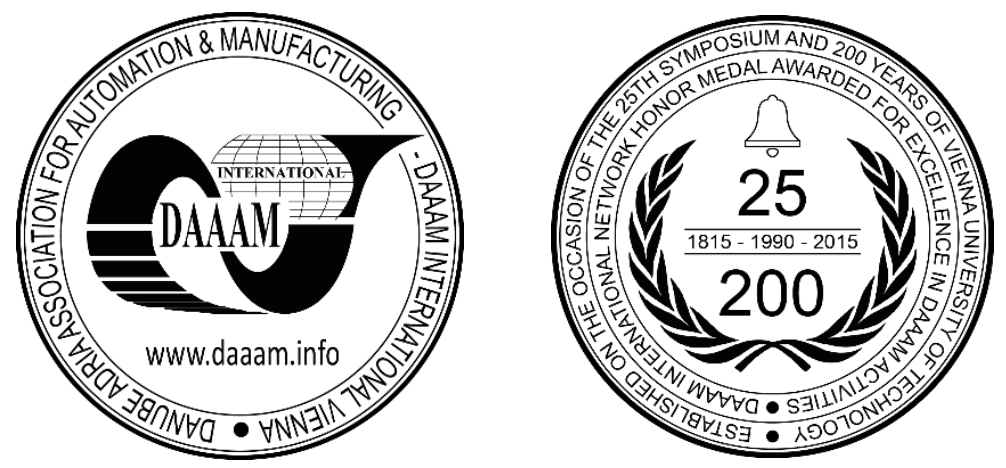

This Publication has to be referred as: Busuladzic, I[brahim]; Horman, I[zet] \& Hajdarevic, S[eid] (2017). Prediction of Wear of Woodworking Tools Depending of the Chip Thickness by Boundary Element Method Simulation, Proceedings of the 28th DAAAM International Symposium, pp.0372-0378, B. Katalinic (Ed.), Published by DAAAM International, ISBN 978-3-902734-11-2, ISSN 1726-9679, Vienna, Austria

DOI: $10.2507 / 28$ th.daaam.proceedings.052

\begin{abstract}
A good quality of produced workpiece in wood industry requires the suitable machines and accompanying tools. Used tools must be constructed from adequate materials and with appropriate geometry depending of technological purpose. Using tools, cutting blade tend to blunt during the production cycle. One of the most important phenomena is the plastic deformation of cutting edge due to high temperature occurrence. In this study we analyse the influence of the chip thickness on temperature occurrence inside the blade. We use the Boundary Element Method (BEM) to analyse the temperature occurrence causing the plastic deformation in material and the blunting of the cutting edge. Different thickness of chips cause the different types of blunting whether the occurrence of craters or rounding of the blade.
\end{abstract}

Keywords: Wood cutting tools; tool wear; temperature occurrence; plastic deformation; boundary element method

\section{Introduction}

When processing wood by mechanical cutting, friction occurs between the separated particles and the front side of the tool, between the rake face of the tool and the cutting surface and internal friction between the particles of the treated material. In order to overcome the frictional forces, the mechanical energy should always be brought to the cutting place. Looking at the energy balance after cutting, it can be concluded that a very small portion of the generated energy is converted into the kinetic energy of the saw, while the bulk of the energy brought is transformed by friction into the heat energy.

Depending of the chip thickness the tool wear will be different. It can be described by the occurrence of craters or rounding of the blade. Plastic deformation caused by high temperature and frictional work between tool and workpiece is one of the most influential factors on tool wear. Experimental studies and different techniques can be used to determine the lifetime of cutting edges. Wear due to temperature is typical limitation of tool life. High temperature of the edge of a tool increases the wear of a tool as heat weakens and softens metals especially above 500$600 \mathrm{C}^{0}$. Sheikh-Ahmad et al. [1] have studied the effects of high temperature on tool wear of cemented tungsten carbide. Certain authors have employed different techniques to measure temperature on cutting tools. Chao et al. [2] have utilized 
infrared thermometer to measure the temperature on lathe tool. Csanady [3] has used sensors inside the tool in bisector line. To decrease the effect of tool heating Gazo et al. [4] have applied techniques of tool cooling using refrigerated air.

Sheikh-Ahmad et al. [5] have employed experimental measurement and Boundary Element Method to study influences of cutting speed on temperature distribution in a wood cutting tool. The Boundary Element Method will be used in this paper to represent the influence of chip thickness on temperature distribution inside the tool causing the plastic deformations. In this study, we use numerical technique to determine the type of tool wear without limitation on the outside or inside of the tool.

\section{Blunting of cutting tools by plastic deformations}

It can be important to determine temperature distribution by numerical means facilitating to develop predictive models of tool wear. When we investigate a process of cutting, we can see that on the edge and on the front surface of the knife, the pressure distribution develops such as shown in figure 1a, while the chip moves with speed $v$ in regards to the knife. From the thermodynamic point of view, the material separation process is seen as a composition of four porters: particle, processing, tool and environment (figure 1b).

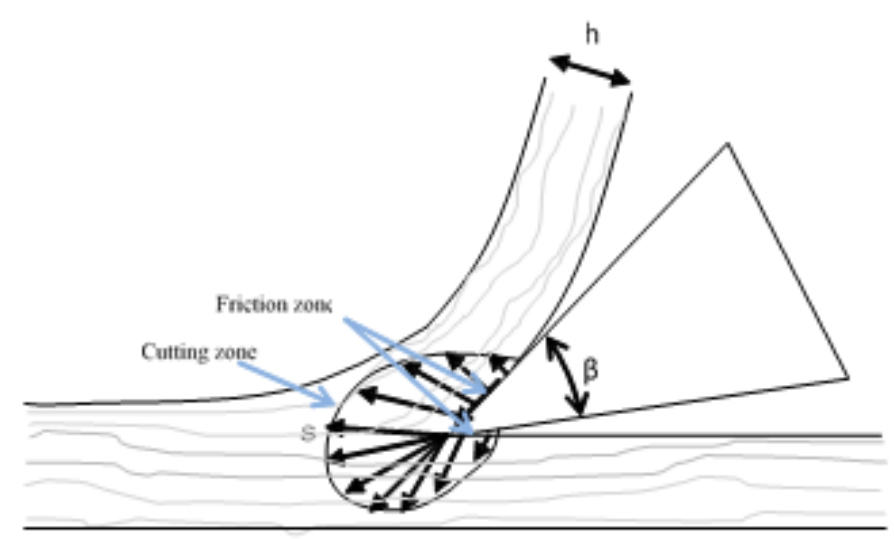

a)

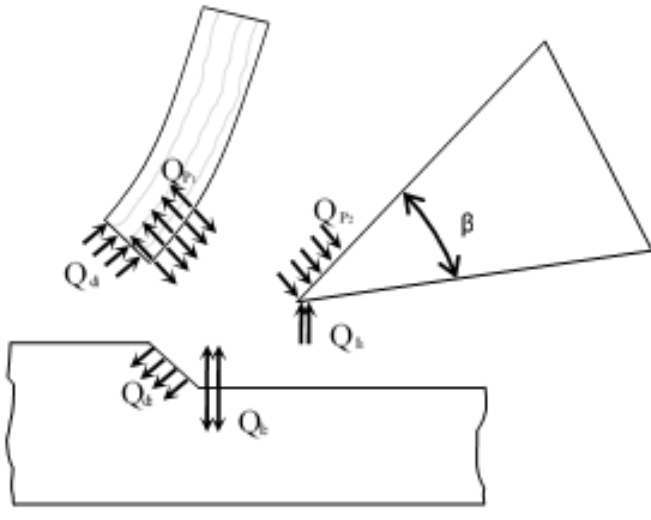

b)

Fig. 1. a) Pressure distribution over cutting process, b) Material separation from thermodynamic - point of view

The theoretical value of the generated heat can be written as [6]:

$$
Q=v \int_{F} \mu \sigma \cdot d f
$$

The amount of heat produced is approximately equal to the work consumed (ignoring the kinetic energy of the chip). Therefore, the amount of heat that is generated in the unit of time and can be written as.

$$
Q \approx F \cdot v
$$

$Q$ is the amount of heat generated in the unit of time, $F$ is the force component on the blade in the direction of the cutting speed, and $v$ the cutting speed.

Taking into account the energy contained in the movement of the chips, for total developed heat the term can be written as:

$$
Q \approx F \cdot v \cdot \alpha_{0}
$$

$\alpha_{0}$ is the factor that takes into account that part of the delivered mechanical energy that is transmitted as kinetic energy to the chip. Usually this part of the energy is neglected.

The resulting heat is the result of external and internal friction.

$$
Q=Q_{l}+Q_{P}+Q_{d}
$$

- $Q_{l}$ is the amount of heat generated in the unit of time by friction between the rake face and cutting surface.

- $Q_{p}$ is the amount of heat generated in the unit of time between the front surface and the chip.

- $Q_{d}$ is the amount of heat generated in the unit of time by internal friction between the chips of the treated material. 
On the front surface, in the unit of time it will be created heat equal to the product of these two quantities.

$$
Q_{p}=F_{t p} \cdot v^{\prime}
$$

$\mathrm{F}_{t p}$ is the frictional force between the separated particle and the front surface of the cutting blade and $\mathrm{v}$ ' is the velocity of the movement of the separated particle. On the front surface of the tool frictional force will be equal to:

$$
F_{t p}=\mu_{p} \cdot F_{N}
$$

$\mathrm{F}_{N}$ is a normal (vertical) force on the tool's front surface, $\mu_{p}$ is the frictional factor between the separated particle and the front surface of the tool.

Comparing the experimental and theoretical values of temperature, it can be shown that only $60 \%$ of theoretical values of frictional forces appear such as effective heat on surface. The temperature generated on the tool body depends on several factors, mainly of; the thickness of the chip, the peripheral velocity and the sharpening angle.

The influence of thickness of the chip on friction and surface temperature causing the tool wear will be the parameter that will be analysed in this study. Numerical-experimental research approach will be adopted in this paper in order to determine the temperature distribution over the whole geometry of the tool in continuous process of cutting wood.

The target of this approach is to deliver a representative amount of the cutting power passing into thermal energy, which takes place in the tool. As this quantity of heat is not known, a priori the inverse technique is adopted in which the heat transfer in the tool is adapted to numerical model until the moment when the temperature in distant areas on the field coincide with the experimental measurements.

Measuring the cutting force one can determine friction coefficient. The previous questions can be answered partly from experimental results and partly from theoretical considerations. If the boundary conditions; the location of the heat flow and intensity, heat transfer on the surface are known, then the differential equation of the heat conductivity can be solved with a computer by numerical simulations. Boundary Element Method can be used to determine the whole temperature field.

The empirical equation describing the temperature can be written as follows [6]:

$$
T=T_{0}+\left(26.6 \rho^{0.8}+1053 \cdot x^{0.8}\right) \frac{v^{0.8}}{\beta^{0.95}}
$$

Csanady [2] has measured the temperature at bisector line of the tool at certain distance from the cutting edge. Extrapolation of the results at the known points in bisector line can give the value of temperature at the edge of tool.

Depending of the chip thickness we have different type of blunting whether craters or rounding of the blade. The equivalent length of the heat inflow on the front face depends primarily on the thickness of the chip and its value, from experimental observations and theoretical considerations, may be taken as:

$$
L=(1,2-1,3) h
$$

In figure 2 we illustrate the process of cutting for thick and thin chip.
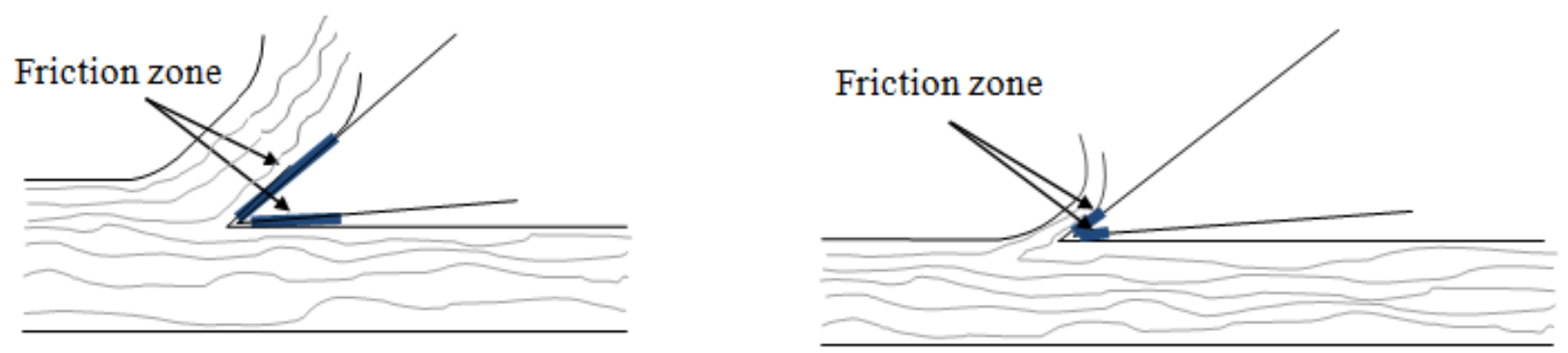

Fig. 2. Process of cutting with thick and thin chip

Increasing the thickness of the chip, the length of the heat inflow on the front face will increase. The chip takes a more curled shape if the chip is thinner and straighter if the chip is thicker. It is one of reasons that the length of heat inflow is longer when the chip is thicker. Also, the force exerted on cutting edge is higher with thicker chip resulting with larger heat generated on the front surface of the cutting tool.

With different thickness of the chip one can differentiate two types of cutting edge wear. In figure 3 we can see the different types of cutting edge wear in function of thickness of the chip and the cutting speed. Working with higher cutting speed the blunting is in form of craters already at a slight thickness. 


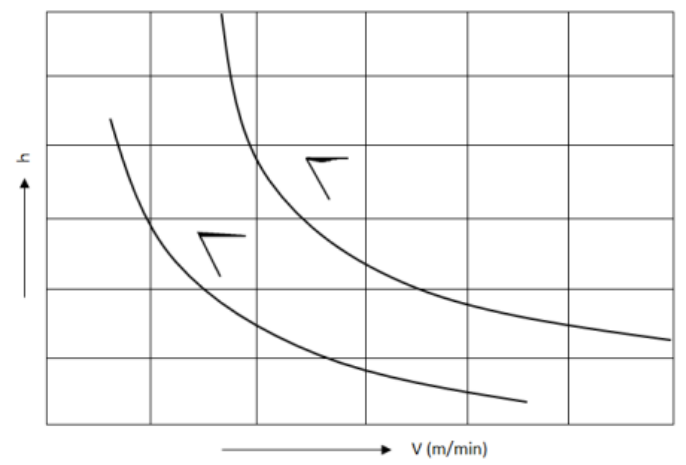

Fig. 3. Different types of tool blunting

Applying the boundary conditions in function of length of the chip in contact with the tool, respectively with cutting edge we change temperature field inside the tool. During the cutting process, thicker chip is longer in contact with tool then thinner ones. These boundary conditions will be applied during the numerical simulation via Boundary Element Method.

\section{Boundary element method - BEM}

Many numerical methods are available to solve thermal models and have been applied to solve thermal problems in cutting tools. In this study we have used the Boundary Element Method (BEM). BEM has the advantages that we profit in our study; the resolution in certain conditions is faster than with other numerical methods and it is easy to get solution in every point anywhere inside the domain.

Here below discretization is made for a simple two dimensional geometry with elements, nodes and internal points (figure 4).

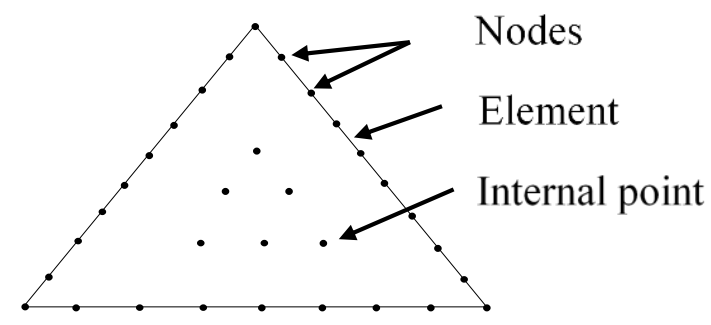

Fig. 4. Example of BEM meshing in 2D

Stationary heat conduction processes are governed by Laplace's equation. The problem is therefore written in general by the following equation:

$$
\forall M \in \Omega, \quad \vec{\nabla}^{2} T(M)=0
$$

With following boundary conditions:

$$
\begin{aligned}
& \forall M \in \Gamma_{T}, \quad T(M)=T_{0} \\
& \forall M \in \Gamma_{\phi}, \quad-\lambda \vec{\nabla} T(M) \cdot \vec{n}=\phi_{0}=-\lambda q_{0}
\end{aligned}
$$

Note $q=\vec{\nabla} T \cdot \vec{n}$, the temperature gradient in the outward normal (and $q^{*}=\vec{\nabla} T^{*} \cdot \vec{n}$ ).

\section{Fundamental Solution}

The fundamental solution $\mathrm{T}^{*}$ satisfies Laplace's equation and represents the field generated by a concentrated unit charge acting at a point ' $i$ '. For an isotropic medium 2D, $\mathrm{T}^{*}$ and $\mathrm{q}^{*}$ are Green functions.

$$
T^{*}=\frac{1}{2 \pi} \ln \frac{1}{r}
$$


$q^{*}=\vec{\nabla} T^{*} \cdot \vec{n}=\frac{d}{2 \pi r^{2}}$

Where $r$ is the distance from the point of collocation $\mathrm{P}$ to the point $\mathrm{M}$ of the considered border $(r=\|r\|$ and $r=P M)$. " $d$ " is the distance of the opposite projection of $\mathrm{r}$ on the outgoing normal $\mathrm{M}(d=-\vec{r} \cdot \vec{n})$. The distances $\mathrm{r}$ and $\mathrm{d}$ are shown in figure 5.

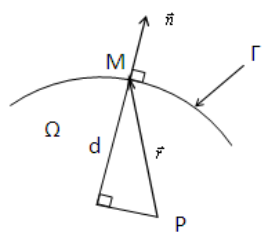

Fig. 5. Geometry definitions

\section{Boundary integral equation}

Using the definition of the Dirac function $\delta p$ we obtain the integral formulation on the boundary. Applying this equation to a collocation point $i$ (or the boundary area) [7], we have:

$$
c_{i} T_{i}+\int_{\Gamma} T q^{*} d \Gamma=\int_{\Gamma} q T^{*} d \Gamma
$$

The most important features of boundary elements, for a 2D problem, however is that it only requires discretization of the contour rather than the surface. The calculation of integrals $\Gamma$ of this equation is equivalent to a $1 \mathrm{D}$ integrals on the border. It is divided into elements which may be chosen from several types according to the degree of interpolation and continuity. In our simulations we have used the constant elements. The integral equation is initially applied to the nodes of the boundary, which allows to obtain solutions at these points. In a second step, the equation can be applied to points within domain called internal points.

If boundary integral equation is applied to the " $i$ '" collocation point located within the domain, the form $c_{i}$ factor takes the value 1 .

$$
T_{i}=\int_{\Gamma} q T^{*} d \Gamma-\int_{\Gamma} T q^{*} d \Gamma
$$

\section{Results and discussion}

For first calculation we have validated our simulation on bisector line with experimental results. In this study we have taken a cutting tool to figure out the temperature distribution in this type of tools. The sharpening angle $\beta$ of the tooth in the first simulation is $45^{\circ}$. We take 28 elements and 9 internal points placed on bisector line. Comparing numerical with experimental results we have a good accordance (figure 6).

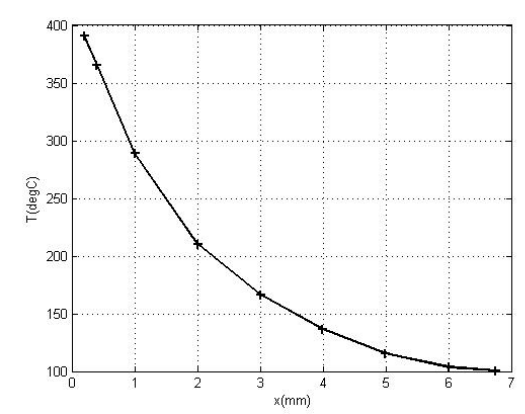

Fig. 6. Validation of BEM simulation

In next instance we have made a simulation for the case of simple blade applying two different thicknesses of chip. Those different thicknesses cause different types of blade blunting. Simulation is made with 28 elements and 18 internal points. The mesh of contour (1D) is finer near the cutting edge. In this section we place more internal points in denser spacing. First case with thin chip shows that we have occurrence of high temperature field close to the cutting edge causing the plastic deformations of the edge (figure 7.a.). This is the situation when we will have rounding of the cutting 
edge. The second case, with thicker chip shows that the plastic deformation is displaced with temperature field a little bit from cutting edge and that will cause deterioration of front surface forming shape of craters (figure 7.b.). This amount of displacement is correlated to the thickness of the chip and certainly does not just depend of it but also of other factors (material of tool, parameters of productions, main sped etc.) [8].

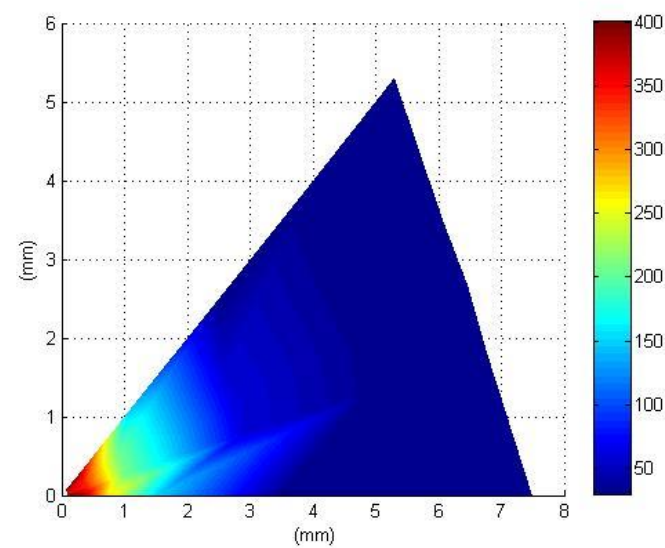

a)

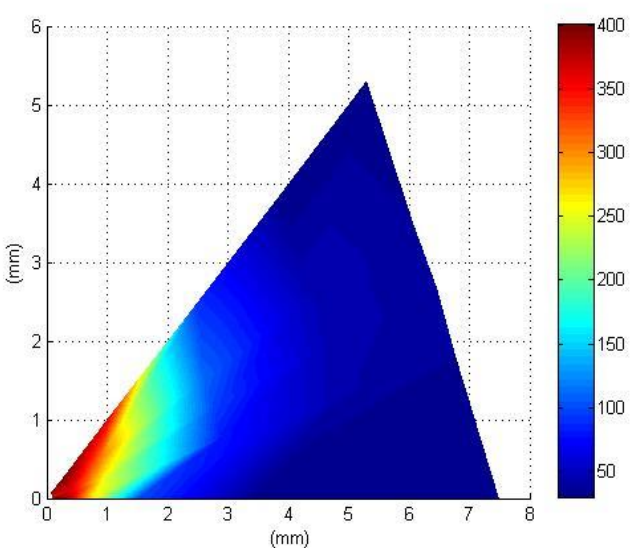

b)

Fig. 7. Temperature distribution for thin a) and thick b) chips

For the real case we take the geometry of the lathe tool on high speed turning. This case is also studied in 2D. The geometry of tool is shown in figure 8 . The sharpening angle of this tool is $60^{\circ}$. To make this simulation by BEM we took 35 elements and 63 internal points, shown in figure 8 .In figure 9 we have shown the simulation for temperature distribution, processing the workpiece with thin a) and thick b) chip. Near the cutting edge we have generate finer mesh of the contour and we have concentrated dense number of internal points to have representative results in isographs. We applied the boundary conditions similar to the previous case.

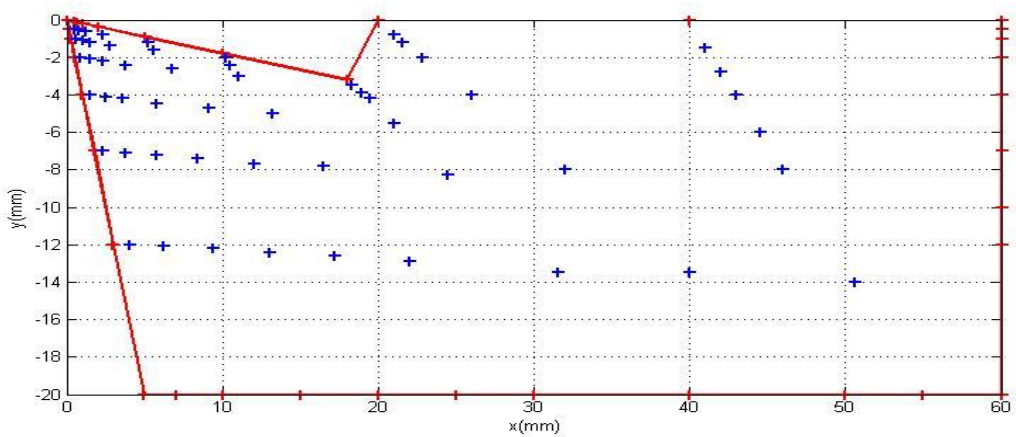

Fig. 8. Mesh of the contour of the lathe tool with internal points

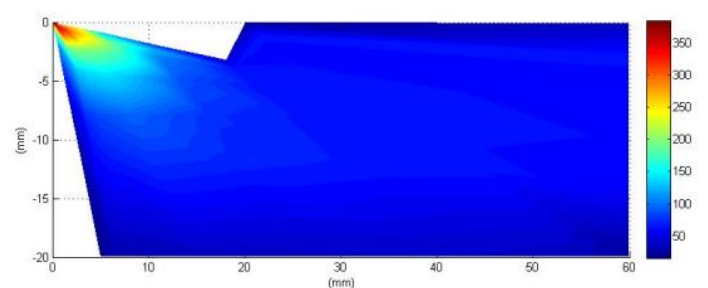

a)

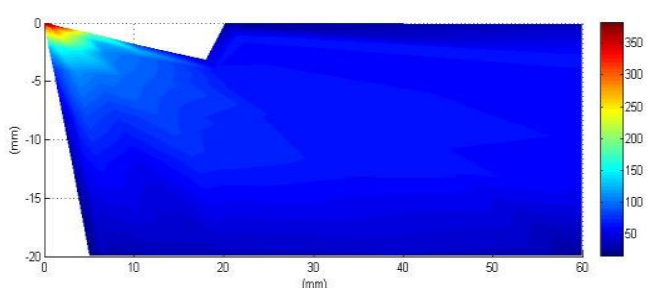

b)

Fig. 9. Temperature distribution for lathe tool for thin a) and thick b) chips

Knowing the thickness of the chip during the process, and helping us with the numerical simulations we can predict the way of cutting edge blunting, whether the occurrence of craters or rounding of the blade. In case of lathe tool we get the similar results such as in the case of simple cutting edge. The most interested regions to observe is near the cutting edge because the temperatures are quasi constants at the rest of geometry and quietly low to influence the tool behaviour. For that reason, the small exchangeable blades are usually used instead of complete tool. 


\section{Conclusion}

Taking different technological conditions of cutting especially with different thickness we have different type of tool blunting. Experimental measurements inside the tool give us inputs to get the numerical solution over whole domain. This inverse technique can be useful to get rapid solution of temperature distribution inside the domain and on the surface of the cutting tool. Here, we use numerical tool via BEM simulation to have very close idea about the way of the tool blunting. Blunting of the tool is directly in relation with plastic deformations respectively with temperature increase. With thicker chip, the chip is straighter and longer in contact with front surface exerting higher force. This force produces immediately larger quantity of heat that is transmitted to the tool. Working with given main speed and thickness under certain value we get the blunting of the tool in the form of rounding of cutting edge. Above certain value of chip thickness we get the blunting in form of craters on front surface and rake face of the tool. The blunting will be pronounced on front surface of the tool. Of course, the technological conditions of cutting depend of other factors that can influence the cutting process such as ability of machines, material of tool, quality of product etc.

Mostly, the thickness of the chip depends of the required quality of wood cutting. Depending of the thickness of the chip, by BEM solution, we can determine the critical zone of tool wear. To decrease the tool wear, one solution is to cool this critical zone by refrigerated air and to use the coatings with good frictional characteristics.

\section{References}

[1] Sheikh-Ahmad, J. Y., Bailey, J. A., (1999). High-temperature Wear of Cemented Tungsten Carbide Tools While Machining Particleboard and Fiberboard, Journal of Wood Science, Vol. 45, No. 6, pp. 445-455, ISSN: 1435-0211

[2] Chao, L. \& Xing, A., (2010). Study on Cutting Temperature and Wear Mechanism of Tool in High Speed Turning Superalloy GH2132, International Conference on Computing, Control and Industrial Engineering, Vol. 2, pp. 327330, Wuhan, DOI:10.1109/CCIE.2010.199

[3] Csanady, E. (2007). Thermal loading in wood cutting tools, Proceedings of Third International Symposium on Wood Machining, ISBN 987-2-88074-725-1, pp. 151-154, Presses polytechniques et universitaire romandes, Lausanne

[4] Gazo, R., Gisip J. \& Stewart H. A. (2011). Reducing tool wear by cryogenic treatment and cooling with refrigerated air when processing medium density fibreboard, In: Wood Machining, J. Paulo Davim), (Ed.), 28 (83-111), ISTE, ISBN 978-1-84821-315-9, London

[5] Sheikh-Ahmad, J. Y., Lewandowski, C. M., Bailey, J. A. \& Stewart, J. S., (2003). Experimental and Numerical Method for Determining Temperature Distribution in a Wood Cutting Tool, Experimental Heat Transfer, Vol.16, No. 4, pp. 255-271, ISSN: 0891-6152, DOI: 10.1080/08916150390223092

[6] Csanady, E. \& Magoss, E. (2013). Mechanics of Wood Machining, Springer, ISBN 978-3-642-29954-4, Berlin Heidelberg

[7] Brebbia, C.A. \& Dominguez, J. (1992). Boundary Elements an Introductory Course, WIT Pess/Computational Mechanics Publications, ISBN 185312160 6, Southampton

[8] Horman, I., Busuladžić, I. \& Azemović, E. (2014). Temperature Influence on Wear Characteristics and Blunting of the Tool in Continuous Wood Cutting Process, Procedia Engineering, Vol. 69, Katalinic, B., pp. 133-140, DOI.org/10.1016/j.proeng.2014.02.213. 